\title{
Nonlocal reductions of the Ablowitz-Ladik equation
}

\author{
Georgi G. Grahovski ${ }^{1}$, Amal J Mohammed ${ }^{2}$ and Hadi Susanto ${ }^{3}$ \\ Department of Mathematical Sciences, University of Essex, Wivenhoe Park, Colchester, UK
}

\begin{abstract}
The purpose of the present paper is to develop the inverse scattering transform for the nonlocal semi-discrete nonlinear Schrödinger equation (known as Ablowitz-Ladik equation) with $\mathcal{P} \mathcal{T}$-symmetry proposed in [10]. This includes: the eigenfunctions (Jost solutions) of the associated Lax pair, the scattering data and the fundamental analytic solutions. In addition, the paper studies the spectral properties of the associated discrete Lax operator. Based on the formulated (additive) Riemann-Hilbert problem, the 1- and 2-soliton solutions for the nonlocal Ablowitz-Ladik equation are derived. Finally, the completeness relation for the associated Jost solutions is proved. Based on this, the expansion formula over the complete set of Jost solutions is derived. This will allow one to interpret the inverse scattering transform as a generalised Fourier transform.
\end{abstract}

\section{Introduction}

Completely integrable infinite-dimensional systems are a subject of a constant interest and a large amount of investigations in different areas of Mathematics and Physics over almost the last five decades $[23,40,59]$ and appear in a wide range of applications - from differential geometry to classical and quantum field theory, fluid mechanics and optics.

A special class of completely integrable infinite-dimensional systems is the class of PDEs integrable by inverse scattering method (ISM) [23,59]. The nonlinear Schrödinger (NLS) equation

$$
\mathrm{i} q_{t}+q_{x x}+2\left|q^{2}\right| q=0, \quad q=q(x, t),
$$

appeared at the very early stage of the development of the ISM $[23,59]$ as one of the classical examples of integrable equations by the ISM and has attracted a significant attention of the scientific community $[19,25,63]$. It appears as an universal model for weakly nonlinear dispersive waves, nonlinear optics and plasma physics [5].

The NLS model has been generalised in several directions. The first one is to consider multi-component generalisations. The first multi-component/vector generalisation of (1) was proposed by S. V. Manakov in 1974 (see [59])

$$
i \mathbf{v}_{t}+\mathbf{v}_{x x}+2\left(\mathbf{v}^{\dagger}, \mathbf{v}\right) \mathbf{v}=0, \quad \mathbf{v}=\mathbf{v}(x, t) .
$$

Here $\mathbf{v}$ is an $n$-component complex-valued vector and $(\cdot, \cdot)$ is the standard scalar product. It is again integrable by the ISM $[5,23,40,59]$. The two component VNLS equation (known as the Manakov model) appears in studies of electromagnetic waves in optical media. Another direction, motivated by the applications of the differential geometric and Lie algebraic methods to soliton type equations $[28,29,32,33,41,43,50]$ (for a detailed review see e.g. [40]) has lead to

\footnotetext{
${ }^{1}$ E-mail: grah@essex.ac.uk

${ }^{2}$ E-mail: ajmoha@essex.ac.uk

${ }^{3}$ E-mail: hsusanto@essex.ac.uk
} 
the discovery of a close relationship between the multi-component (matrix) NLS equations and the homogeneous and symmetric spaces [43].

The first integrable discretisation of the NLS equation (1) was proposed by M. J. Ablowitz and A. Ladik and has the form $[2,3,4]$ :

$$
\mathrm{i} Q_{n, t}=\frac{1}{h^{2}}\left(Q_{n+1}-2 Q_{n+1}+Q_{n-1}\right) \pm\left|Q_{n}\right|^{2}\left(Q_{n+1}+Q_{n-1}\right),
$$

It is a differential-difference or semi-discrete equation (discrete in space and continuous in time), and is in fact a $O\left(h^{2}\right)$ finite difference approximation of (1). The corresponding scattering problem is usually referred to as the Ablowitz-Ladik (AL) scattering problem [5, 6, 7, 17, 34, $35,36,37]$. The equation (3) has also a number of physical applications: it describes the dynamics of anharmonic lattices [56], self-trapping on a dimer [45], various types of Heisenberg spin chains $[44,51]$ and so on. Later on, various discretisations of the NLS models were studied [8,18,19,53,60,61,62] including perturbation effects [21, 22].

Recently, in [9,39] was proposed a nonlocal integrable equation of nonlinear Schrödinger type with $\mathcal{P} \mathcal{T}$-symmetry, due to the invariance of the so-called self-induced potential $V(x, t)=$ $\psi(x, t) \psi^{*}(-x,-t)$ under the combined action of parity and time reversal symmetry. In the same paper, the 1-soliton solution for this model is derived and it was shown that it develops singularities in finite time. Soon after this, nonlocal $\mathcal{P} \mathcal{T}$-symmetric generalisations are found for the Ablowitz-Ladik model in [10]. All these models are integrable by the Inverse Scattering Method (ISM) [11].

The nonlocal reductions of the NLS (1) and the Ablowitz-Ladik equation (3) are of particular interest in regards to applications in $\mathcal{P} \mathcal{T}$-symmetric optics, especially in developing of theory of electromagnetic waves in artificial heterogenic media $[1,14]$. For an up-to-date review, see for example [24,64].

The initial interest in such systems was motivated by quantum mechanics $[15,48]$. In [15] it was shown that quantum systems with a non-hermitian Hamiltonian admit states with real eigenvalues, i.e. the hermiticity of the Hamiltonian is not a necessary condition to have real spectrum. Using such Hamiltonians one can build up new quantum mechanics $[15,16,48,49]$. Starting point is the fact that in the case of a non-Hermitian Hamiltonian with real spectrum, the modulus of the wave function for the eigenstates is time-independent even in the case of complex potentials.

Historically the first pseudo-hermittian hamiltonian with real spectrum is the $\mathcal{P} \mathcal{T}$-symmetric one in [15]. Pseudo-hermiticity here means that the Hamiltonian $\mathcal{H}$ commutes with the operators of spatial reflection $\mathcal{P}$ and time reversal $\mathcal{T}$ : $\mathcal{P} \mathcal{T H}=\mathcal{H} \mathcal{H} \mathcal{T}$. The action of these operators is defined as follows: $\mathcal{P}: x \rightarrow-x$ and $\mathcal{T}: t \rightarrow-t$. Supposing that the wave function is a scalar, this leads to the following action of the operator of spatial reflection on the space of states: $\mathcal{P} \psi(x, t)=\psi(-x, t)$ and $\mathcal{T} \psi(x, t)=\psi^{*}(x,-t)$. As a result, the Hamiltonian and the wave function are $\mathcal{P} \mathcal{T}$-symmetric, if $\mathcal{H}(x, t)=\mathcal{H}^{*}(-x,-t)$ and $\psi(x, t)=\psi^{*}(-x,-t)$. Here we used also, that the parity operator $\mathcal{P}$ is linear and unitary while the time reversal operator $\mathcal{T}$ is anti-linear and anti-unitary.

The action $\mathcal{P}$ and $\mathcal{T}$ operators on the Hamiltonian induces an action on the associated scattering problem (see (6) below) and to its potential (8):

$$
\mathcal{P} Q_{n}(t)=Q_{-n}(t), \quad \mathcal{T} Q_{n}(t)=Q_{n}^{*}(-t) .
$$

This leads to the following reduction (symmetry) condition

$$
Q_{n}^{-}(t)= \pm\left(Q^{+}\right)_{-n}^{*}(t)
$$


As a result, one gets the nonlocal Ablowinz-Ladik equation with $\mathcal{P} \mathcal{T}$ symmetry, proposed in [10]:

$$
\mathrm{i} Q_{n, \tau}^{+}=\left(Q_{n+1}^{+}-2 Q_{n}^{+}+Q_{n-1}^{+}\right)-\epsilon Q_{n}^{+}\left(Q^{+}\right)_{-n}^{*}\left(Q_{n+1}^{+}+Q_{n-1}^{+}\right), \quad \epsilon= \pm 1 .
$$

The purpose of this paper is to develop the inverse scattering transform for (5), to study the spectral properties of the associated Lax operators (6) and (7) and to derive 1- and 2-soliton solutions.

The structure of the present paper is as follows: in Section 2 we briefly outline the structure of the semi-discrete Lax representation, the corresponding semi-discrete (differential-difference) zero-curvature equation and the resulting differential-difference equations. In Section 3 we present the direct scattering transform for the nonlocal Ablowitz-Ladik equation. This includes: the Jost solutions, the scattering matrix and the scattering data, and the fundamental analytic solutions. In Section 4 we formulate a Riemann-Hilbert problem (in additive form) for the fundamental analytic solutions on the continuous spectrum of the discrete Lax operator. Based on this, we derive the 1- and 2-soliton solutions of (5). finally, in Section 5 we describe the spectral properties of the discrete Lax operator, prove the completeness relation for the Jost solutions and derive expansion formula over the complete set of Jost solutions.

\section{Preliminaries}

The starting point here is the semi-discrete analogue of the Lax (or zero-curvature) representation: the initial nonlinear evolutionary equation (3) can be represented as a compatibility condition of two linear systems:

$$
\begin{aligned}
\Psi_{n+1}(z, t) & =L_{n}(z, t) \Psi_{n}(z, t) ; \quad n \in \mathbb{N}, \\
\Psi_{n, t}(z, t) & =M_{n}(z, t) \Psi_{n}(z, t),
\end{aligned}
$$

where

$$
L_{n}(z, t)=\left(\begin{array}{cc}
z & Q_{n}^{+}(t) \\
Q_{n}^{-}(t) & z^{-1}
\end{array}\right)
$$

is an element of the Lie group $S L(2, \mathbb{C})$ and $M_{n}(z, t)$ is an element of the corresponding Lie algebra $\operatorname{sl}(2, \mathbb{C})$. Here we also assume that $Q_{n}^{ \pm}(t)$ are complex-valued functions, satisfying

$\sum_{n=-\infty}^{\infty}<\infty$. The compatibility condition (i.e. the semi-discrete analogue of the zero-curvature representation) of (6) and (7) takes the form:

$$
M_{n+1}=L_{n, t} L_{n}^{-1}+L_{n} M_{n} L_{n}^{-1},
$$

where the operator $M_{n}$ takes the form $M_{n}(z, t)=V_{n}(z, t)+\Omega(z)$, with

$$
V_{n}(z, t)=\mathrm{i}\left(\begin{array}{cc}
Q_{n}^{+}\left(Q_{1-n}^{+}\right)^{*} & z^{-1} Q_{n-1}^{+}+z Q_{n}^{+} \\
\left(z^{-1}\left(Q_{-n}^{+}\right)^{*}-z\left(Q_{1-n}^{+}\right)^{*}\right) & -\left(Q_{-n}^{+}\right)^{*} Q_{n-1}^{+}
\end{array}\right), \quad \Omega=\frac{\mathrm{i}}{2}\left(z-z^{-1}\right)^{2} \sigma_{3} .
$$

The equation (3) together with appropriate boundary conditions is integrable by the inverse scattering method $[2,3,4]$. In addition equation (3) is one of the members of the integrable hierarchy associated to the spectral problem (6).

The discrete compatibility condition (9) results in the following system of differentialdifference equations (without using the involution):

$$
\begin{aligned}
\mathrm{i} Q_{n, \tau}^{+} & =\left(Q_{n+1}^{+}-2 Q_{n}^{+}+Q_{n-1}^{+}\right)-Q_{n}^{+} Q_{n}^{-}\left(Q_{n+1}^{+}+Q_{n-1}^{+}\right) \\
-\mathrm{i} Q_{n, \tau}^{-} & =\left(Q_{n+1}^{-}-2 Q_{n}^{-}+Q_{n-1}^{-}\right)-Q_{n}^{-} Q_{n}^{+}\left(Q_{n+1}^{-}+Q_{n-1}^{-}\right)
\end{aligned}
$$


If one imposes the standard symmetry condition (involution) $Q_{n}^{-}(t)=\epsilon\left(Q_{n}^{+}(t)\right)^{*}$, then one will get (3). If one sets the nonlocal involution $Q_{n}^{-}(t)=\epsilon\left(Q_{-n}^{+}(t)\right)^{*}$, then this will result in (5).

Finally, we note that the Ablowitz-Ladik Lax operator $L_{n}(z)$ can be transformed into a spectral (eigenvalue) problem $\mathcal{L}_{n}(z) \Psi_{n}(z)=0$, where

$$
\mathcal{L}_{n}(z)=\left(\begin{array}{cc}
D_{+} & 0 \\
0 & D_{-}
\end{array}\right)+U_{n}-z \mathbb{I}, \quad U_{n}=\left(\begin{array}{cc}
0 & Q_{n}^{+} \\
Q_{n-1}^{-} & 0
\end{array}\right) .
$$

Here, $D_{ \pm}$are the shift operators $D_{ \pm} \Psi_{n}(z)=\Psi_{n \pm 1}(z)$ and $\mathbb{I}$ is the $(2 \times 2)$ identity matrix [34,35].

The generic nonlocal involution for the system (8) can be written in the form

$$
\mathbf{C}\left[L_{n}(z)\right]:=B L_{-n}\left(z^{*}\right)^{\dagger} B^{-1}=L_{n}(z)
$$

where $B$ is an automorphism of the Lie group $S L(2, \mathbb{C})$. The particular choice $B=\operatorname{diag}(1,-1)$ leads to $(5)$.

\section{Direct scattering transform}

\subsection{Jost solutions and scattering data}

The eigenfunctions of $L_{n}(z)$ and $M_{n}(z)$ of (6)-(7) are defined by their asymptotics (the so-called Jost solutions) for $|n| \rightarrow \infty$ (see e.g. [23, 40,59]):

$$
\psi_{n}(z) \longrightarrow\left(\begin{array}{cc}
z^{n} & 0 \\
0 & z^{-n}
\end{array}\right), \quad \text { as } \quad n \rightarrow+\infty, \quad \phi_{n}(z) \longrightarrow\left(\begin{array}{cc}
z^{n} & 0 \\
0 & z^{-n}
\end{array}\right), \quad \text { as } \quad n \rightarrow-\infty .
$$

Along with the standard Jost solutions, one can define "renormalised" Jost solutions from the functions $\psi_{n}(z), \phi_{n}(z)$, that satisfy the scattering problem (6)

$$
\xi_{n}(z)=\psi_{n}(z) \mathbf{Z}^{-n}, \quad \varphi_{n}(z)=\phi_{n}(z) \mathbf{Z}^{-n}
$$

where $\mathbf{Z}=\left(\begin{array}{cc}z & 0 \\ 0 & z^{-1}\end{array}\right)$, and the eigenfunctions $\xi_{n}, \varphi_{n}$ are solution of the difference equations respectively

$$
\xi_{n+1}=\left(\mathbf{Z}+\tilde{\mathbf{Q}}_{n}\right) \xi_{n} \mathbf{Z}^{-1}, \quad \varphi_{n+1}=\left(\mathbf{Z}+\tilde{\mathbf{Q}}_{n}\right) \varphi_{n} \mathbf{Z}^{-1}
$$

where $\tilde{\mathbf{Q}}_{n}=\left(\begin{array}{cc}0 & Q_{n}^{+} \\ Q_{n}^{-} & 0\end{array}\right)$, with the canonical boundary conditions

$$
\lim _{x \rightarrow \infty} \xi_{n}(z)=\mathbb{I}, \quad \lim _{x \rightarrow-\infty} \varphi_{n}(z)=\mathbb{I} .
$$

The two Jost solutions $\phi_{n}(z)$ and $\psi_{n}(z)$ are related by the scattering matrix:

$$
\phi_{n}(z)=\psi_{n}(z) T, \quad T=\left(\begin{array}{cc}
a^{+}(z) & -b^{-}(z) \\
b^{+}(z) & a^{-}(z)
\end{array}\right)
$$

The nonlocal involution (13) imposes symmetry condition as Jost solutions associated with (8), and will be

$$
\mathbf{C}\left(\psi_{-n}^{\dagger}\left((z)^{*}, t\right)\right)=B \psi_{-n}^{\dagger}\left((z)^{*}, t\right) B^{-1}=\phi_{n}(z, t)
$$




\subsection{Fundamental Analytic Solutions}

Important tools for reducing the ISP to a Riemann-Hilbert problem (RHP) are the fundamental analytic solution (FAS) $\chi^{ \pm}(x, t, \lambda)$. Their construction is based on the Gauss decomposition of $T(\lambda, t)$, see $[26,27,59]$ :

$$
\begin{aligned}
& \chi_{n}^{+}(z)=\psi_{n}(z) T^{-}(z)=\phi_{n}(z) S^{+}(z), \\
& \chi_{n}^{-}(z)=\psi_{n}(z) T^{+}(z)=\phi_{n}(z) S^{-}(z),
\end{aligned}
$$

where

$$
\begin{array}{rlrl}
T^{-}(z) & =\left(\begin{array}{cc}
a^{+}(z) & 0 \\
b^{+}(z) & 1
\end{array}\right), & T^{+}(z)=\left(\begin{array}{cc}
1 & -b^{-}(z) \\
0 & a^{-}(z)
\end{array}\right), \\
S^{+}(z)=\left(\begin{array}{cc}
1 & \beta^{-}(z) \\
0 & \alpha^{+}(z)
\end{array}\right), & S^{-}(z)=\left(\begin{array}{cc}
\alpha^{-}(z) & 0 \\
-\beta^{+}(z) & 1
\end{array}\right)
\end{array}
$$

are the factors in the Gauss decomposition of the associated scattering matrix $T(z)$ :

$$
T(z)=T^{-}(z) \hat{S}^{+}(z)=T^{+}(z) \hat{S}^{-}(z),
$$

and are expressed in terms of the matrix elements of the scattering matrix $T(z)$ and its inverse

$$
\hat{T}(z)=\left(\begin{array}{cc}
\alpha^{-}(z) & \beta^{-}(z) \\
-\beta^{+}(z) & \alpha^{+}(z)
\end{array}\right)
$$

This construction ensures that $\xi_{n}^{ \pm}(z)$ are analytic functions of $z$ for $z \in \Omega_{ \pm}$.

On the unit circle $|z|=1$ (i.e., on the continuous spectrum of $L_{n}(z)$ ), the two FAS are linearly dependent:

$$
\tilde{\chi}_{n}^{+}(z)-\tilde{\chi}_{n}^{-}(z)=\tilde{\chi}_{n}^{-}(z) \mathbf{G}_{n}(z) \quad|z|=1,
$$

where the sewing function $G_{n}(z, t)$ can be expressed in terms of $\rho^{ \pm}(t, z)$ :

$$
\mathbf{G}_{n}(z, t)=\left(\begin{array}{cc}
\rho^{+} \rho^{-} & z^{2 n} \rho^{-} \\
z^{-2 n} \rho^{+} & 0
\end{array}\right), \quad \tilde{\chi}_{n}^{-}(z) \rightarrow \mathbb{1} \quad \text { as }|z| \rightarrow \infty .
$$

The independent matrix elements of $G_{n}(z, t)$, together with the discrete spectrum of $L_{n}(z)$ form up the minimal set of scattering data of $L_{n}$.

The nonlocal involution (13) imposes symmetry condition on the FAS and the scattering matrix as follows:

$$
\mathbf{C}\left(\chi_{-n}^{\{-, \dagger\}}\left((z)^{*}, t\right)\right)=B \chi_{-n}^{\{-, \dagger\}}\left((z)^{*}, t\right) B^{-1}=\chi_{n}^{+}(z, t)
$$

and

$$
\mathbf{C}\left(T^{\dagger}\left((z)^{*}, t\right)\right)=B T^{\dagger}\left((z)^{*}, t\right) B^{-1}
$$

As a result, we obtain

$$
a^{ \pm}(z, t)=\left(a^{ \pm}\left(z^{*}, t\right)\right)^{*}, \quad b^{ \pm}(z, t)=\epsilon\left(b^{\mp}\left(z^{*}, t\right)\right)^{*}
$$

\subsection{Asymptotic Behavior of FAS}

Until the end of this section, we will presume that the involution (13) holds true. 


\subsubsection{Asymptotic Behavior of FAS for $|z|=1$}

The discrete scattering problem (6) can possess discrete eigenvalues. This can occur when $a^{ \pm}\left(z_{j}\right)=0$ for some $z_{j}$. Here we will assume that this cannot happen on the continuous spectrum. On the zeroes $z_{j}$ of $a^{ \pm}(z)$, the two Jost solutions become proportional:

$$
\varphi_{n}^{ \pm}\left(z_{j}\right)= \pm b_{j}^{ \pm} z_{j}^{\mp 2 n} \xi_{n}^{ \pm}\left(z_{j}\right) .
$$

Assume that $a^{+}(z)$ has $S$ simple zeros $\left\{z_{j}:\left|z_{j}\right|>1\right\}_{j=1}^{S}$ and $a^{-}(z)$ has $S$ simple zeros $\left\{z_{j}:\left|z_{j}\right|<1\right\}_{j=1}^{S}$, i.e., the number of zeroes inside the unit circle is equal to the number of zeroes outside the unit circle. Then, by equations (28):

$$
\operatorname{Res}\left(\tilde{\varphi}_{n}^{ \pm}, z_{j}^{ \pm}\right)=\frac{\varphi_{n}^{ \pm}\left(z_{j}^{ \pm}\right)}{\dot{a}^{ \pm}\left(z_{j}^{ \pm}\right)}= \pm \frac{b_{j}^{ \pm}\left(z_{j}^{ \pm}\right)^{\mp 2 n} \xi_{n}^{ \pm}}{\dot{a}^{ \pm}\left(z_{j}^{ \pm}\right)}= \pm\left(z_{j}^{ \pm}\right)^{\mp 2 n} C_{j}^{ \pm} \xi_{n}^{ \pm}\left(z_{j}^{ \pm}\right),
$$

where we have denoted the norming constants as $C_{j}^{ \pm}$.

\subsubsection{Asymptotic Behavior of FAS for $|z| \rightarrow \infty$ and $|z| \rightarrow 0$}

Then the Laurent expansions of FASs $\chi_{n}^{ \pm}(z)$ have the following form

$$
\begin{aligned}
& \chi_{n}^{+}(z)=\left(\begin{array}{cc}
1+O\left(z^{-2}, \text { even }\right) & c_{n}^{-1} z^{-1} Q_{n}^{+}+O\left(z^{-3}, \text { odd }\right) \\
z^{-1} Q_{n-1}^{-}+O\left(z^{-3}, \text { odd }\right) & c_{n}^{-1}+O\left(z^{-2}, \text { even }\right)
\end{array}\right) \quad \text { as }|z| \rightarrow \infty, \\
& \chi_{n}^{-}(z)=\left(\begin{array}{cc}
c_{n}^{-1}+O\left(z^{2}, \text { even }\right) & z Q_{n-1}^{+}+O\left(z^{3}, \text { odd }\right) \\
c_{n}^{-1} z Q_{n}^{-}+O\left(z^{3}, \text { odd }\right) & 1+O\left(z^{2}, \text { even }\right)
\end{array}\right) \quad \text { around } \quad z=0 .
\end{aligned}
$$

From the analytic properties of the eigenfunctions of $L_{n}(z)$, it follows that $a^{+}(z)$ has an analytic extension in the region $|z| \rightarrow \infty$ :

$$
W\left(\varphi_{n}^{+}, \xi_{n}^{+}\right)=W\left(\begin{array}{cc}
1+O\left(z^{-2}, \text { even }\right) & c_{n}^{-1} z^{-1} Q_{n}+O\left(z^{3}, \text { odd }\right) \\
z^{-1} R_{n-1}+O\left(z^{-3}, \text { odd }\right) & c_{n}^{-1}+O\left(z^{-2}, \text { even }\right)
\end{array}\right)
$$

then $\chi_{n}^{+}(z)$ is analytic when $|z| \rightarrow \infty$

$$
a^{+}(z)=1-O\left(z^{-2} \text {, even }\right) \quad \text { as }|z| \rightarrow \infty .
$$

Similar arguments apply in order to find the Laurent series expansions for $a_{n}^{-}(z)$ :

$$
W\left(\xi_{n}^{-}, \varphi_{n}^{-}\right)=W\left(\begin{array}{cc}
c_{n}^{-1}+O\left(z^{2}, \text { even }\right) & z Q_{n-1}+O\left(z^{3}, \text { odd }\right) \\
c_{n}^{-1} z R_{n}+O\left(z^{3}, \text { odd }\right) & 1+O\left(z^{2}, \text { even }\right)
\end{array}\right),
$$

so, $\chi_{n}^{-}(z)$ is analytic around $|z|=0$ and

$$
a^{-}(z)=1-O\left(z^{2} \text {, even }\right) \quad \text { as }|z| \rightarrow 0 .
$$

The scattering coefficients can be written as infinite explicit sums of the eigenfunctions:

$$
\begin{array}{ll}
a^{+}(z)=1+\sum_{k=-\infty}^{+\infty} z^{-1} Q_{k}^{+} \varphi_{k}^{(2),+}, & b^{+}(z)=\sum_{k=-\infty}^{+\infty} z^{2 k-1} Q_{k}^{-} \varphi_{k}^{(1),+}, \\
a^{-}(z)=1+\sum_{k=-\infty}^{+\infty} z Q_{k}^{-} \varphi_{k}^{(1),-}, & b^{-}(z)=-\sum_{k=-\infty}^{+\infty} z^{-2 k+1} Q_{k}^{+} \varphi_{k}^{(2),-},
\end{array}
$$




\section{The Riemann-Hilbert Problem and Soliton Solutions}

It is well known that the inverse scattering transform for the Lax operator $L_{n}(z)$ can be reduced to a Riemann-Hilbert boundary value problem on the complex plane. The contour, where the values of the analytic functions are specified is the continuous spectrum of $L_{n}(z)(6)$ - in the case of the Ablowitz-Ladik equation it is the unit circle $|z|=1$. If the Lax operator $L_{n}(z)$ has discrete eigenvalues, then the resulting Riemann-Hilbert problem (RHP) is of singular type. Here we will restrict ourselves to the so-called balanced RHPs - we will consider only problems having equal number of singularities inside and outside the boundary contour, i.e. we will assume that the number of zeroes of $a^{+}(z)$ is equal to the number of zeroes of $a^{-}(z)$. The reflectionless case $b^{ \pm}(z)=0$ will correspond to soliton solutions of (5) with $\epsilon=-1$.

- Symmetries and symmetry reductions

Since the expansions of $a^{ \pm}(z)$ we presented in Section 3.2 contain only even power of $z^{-1}$ and $z$, it follows that if $z_{j}^{ \pm}$are zeroes of $a^{ \pm}(z)$ then $-z_{j}^{ \pm}$are also zeros of $a^{ \pm}(z)$. This implies that

$$
C_{j}^{ \pm}\left(-z_{j}^{ \pm}\right)=\frac{b^{ \pm}\left(-z_{j}^{+}\right)}{\dot{a}^{ \pm}\left(-z_{j}^{ \pm}\right)}=C^{ \pm}\left(z_{j}^{+}\right),
$$

where $b_{j}^{-}=-b_{j}^{+}$, and

$$
\rho^{+}(-z)=-\rho^{+}(z), \quad \rho^{-}(-z)=-\rho^{-}(z) .
$$

The nonlocal involution (13) will act on the reflection coefficient and the normalisation constant producing the following constraint:

$$
C_{j}^{-}\left(z_{j}^{-}\right)=\frac{\epsilon\left(b^{+}\left(z_{j}^{+, *}\right)\right)^{*}}{\left(\dot{a}^{-}\left(z_{j}^{-, *}\right)^{*}\right.}, \quad \rho^{-}(z)=\frac{\epsilon\left(b^{+}\left(z^{*}\right)\right)^{*}}{\left(a^{-}\left(z^{*}\right)\right)^{*}} .
$$

- Case of poles

If the FAS $\tilde{\varphi}_{n}^{ \pm}(z)$ have poles, the method of solution of the RHP requires an extra step involving a contour integration. The starting point is the relations between the eigenfunctions,

$$
\tilde{\varphi}_{n}^{ \pm}=\frac{\varphi_{n}^{ \pm}}{a^{ \pm}}(z)=\xi_{n}^{\mp}(z) \pm z^{\mp 2 n} \rho^{ \pm}(z) \xi_{n}^{ \pm}(z)
$$

We will apply the contour integration method to the following integral representations:

$$
\begin{aligned}
& \partial_{1, n}(z)=\frac{1}{2 \pi \mathrm{i}}\left(\oint_{\gamma^{+}} \frac{\mathrm{d} \omega \varphi_{n}^{+}(\omega)}{(\omega-z) a^{+}(\omega)}-\oint_{\gamma^{-}} \frac{\mathrm{d} \omega \xi_{n}^{-}(\omega)}{(\omega-z)}\right), \\
& \partial_{2, n}(z)=\frac{1}{2 \pi \mathrm{i}}\left(\oint_{\gamma^{+}} \frac{\mathrm{d} \omega \xi_{n}^{+}(\omega)}{(\omega-z)}-\oint_{\gamma^{-}} \frac{\mathrm{d} \omega \varphi_{n}^{-}(\omega)}{(\omega-z) a^{-}(\omega)}\right),
\end{aligned}
$$

where the contours of integration $\gamma_{ \pm}$are depicted in Figure 1. Here we will present the detailed evaluation of one of the integrals $\left(\mathcal{J}_{2, n}\right)$. The other one can be evaluated in a similar manner. 


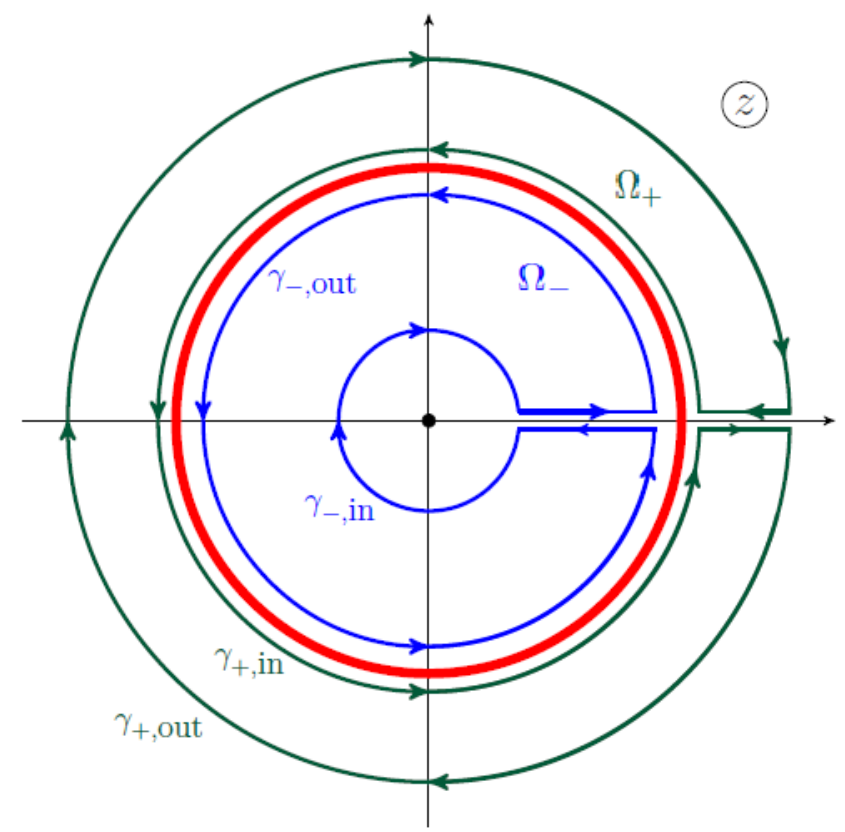

Figure 1: The continuous spectrum $\Omega$ of $L_{n}(z)$ (in red) and the integration contours

Recall that (i) $\frac{1}{a^{+}(z)}$ has simple pole at $z=z_{j}^{+}$; (ii) $\frac{1}{a^{-}(z)}$ has simple pole at $z=z_{j}^{-}$and (iii) $\xi_{n}^{-}, \xi_{n}^{+}$have no poles, and therefore the integrand of the first integral in $\mathcal{J}_{2, n}(z)$ has only a pole at $z=\omega$ and (iv) outside the contour is a negatively oriented, while inside the contour is a positively oriented. Thus when $z \in \Omega_{+}$, we found:

$$
\partial_{2, n}(z)=\xi_{n}^{+}(z)-\sum_{j=1}^{S}\left[\frac{\varphi_{n}^{-}\left(z_{j}^{-}\right)}{\left(z-z_{j}^{-}\right) \dot{a}_{j}^{-}}+\frac{\varphi_{n}^{-}\left(-z_{j}^{-}\right)}{\left(z+z_{j}^{-}\right) \dot{a}_{j}^{-}}\right]
$$

and the integral along the unit circle $\Omega=z \in \mathbb{C}:|z|=1$ is equal to

$$
\frac{1}{2 \pi \mathrm{i}}\left(\oint_{\gamma^{+}} \frac{\mathrm{d} \omega \xi_{n}^{+}(\omega)}{(\omega-z)}-\oint_{\gamma^{-}} \frac{\mathrm{d} \omega \varphi_{n}^{-}(\omega)}{(\omega-z) a^{-}(\omega)}\right)=\frac{1}{2 \pi \mathrm{i}} \oint_{\Omega} \frac{\mathrm{d} \omega}{(\omega-z)} \omega^{2 n} \rho^{-}(z) \xi_{n}^{-}(z) .
$$

If $a^{ \pm}(z)$ have zeros at $z_{j}^{ \pm}$respectively, then one can write

$$
\varphi_{n}^{ \pm}= \pm\left(z_{j}^{ \pm}\right)^{\mp 2 n} b_{j}^{ \pm} \xi_{n}^{ \pm}
$$

and this will lead to the following integral representation for $\xi_{n}^{+}(z)$

$$
\xi_{n}^{+}(z)=\left(\begin{array}{l}
0 \\
1
\end{array}\right)+\frac{1}{2 \pi \mathrm{i}} \oint_{\Omega} \frac{\mathrm{d} \omega}{(\omega-z)} \omega^{2 n} \rho^{-}(\omega) \xi_{n}^{-}(\omega)-\sum_{j=1}^{S} C_{j}^{-}\left(z_{j}^{-}\right)^{2 n}\left[\frac{\xi_{n}^{-}\left(z_{j}^{-}\right)}{\left(z-z_{j}^{-}\right)}+\frac{\xi_{n}^{-}\left(-z_{j}^{-}\right)}{\left(z+z_{j}^{-}\right)}\right] .
$$

In a similar way, one can find an integral representation for $\xi_{n}^{-}(z)$ with $z \in \Omega_{-}$from evaluating the integral $\partial_{1, n}$,

$$
\xi_{n}^{-}(z)=\left(\begin{array}{l}
1 \\
0
\end{array}\right)+\frac{1}{2 \pi \mathrm{i}} \oint_{\Omega} \frac{\mathrm{d} \omega}{(\omega-z)} \omega^{-2 n} \rho^{+}(\omega) \xi_{n}^{+}(\omega)+\sum_{j=1}^{S} C_{j}^{+}\left(z_{j}^{+}\right)^{-2 n}\left[\frac{\xi_{n}^{+}\left(z_{j}^{+}\right)}{\left(z-z_{j}^{+}\right)}+\frac{\xi_{n}^{+}\left(-z_{j}^{+}\right)}{\left(z+z_{j}^{+}\right)}\right] .
$$


The integrands in the integral representations (41b) and (41c) are expressed in terms of $\rho^{ \pm}(z)$ on the continuous spectrum only, while the sums that give the contributions from the discrete spectrum are expressed in terms of the normalisation constants $C_{j}^{ \pm}\left(z_{j}^{ \pm}\right)$. Thus the system of singular integral equations (41) admits unique solution, so the minimal set of scattering data $\mathcal{F}_{1}=\left\{\rho^{+}(z), \rho^{-}(z), z \in|z|=1, z_{j}^{ \pm}, j=1, \ldots S\right\}$ contains all information needed to recover uniquely the Jost solutions $\xi_{n}^{ \pm}(z)$.

\subsection{1-Soliton solution}

In the case where the Lax operator $L_{n}(z)$ comprises proper eigenvalues but $\rho^{+}(z)=\rho^{-}(z)=0$ on the continuous spectrum of $L_{n}(z)$, then (41) reduces to linear system of algebraic equations:

$$
\begin{aligned}
& \xi_{n}^{+}(z)=\left(\begin{array}{l}
0 \\
1
\end{array}\right)-\sum_{j=1}^{S} C_{j}^{-}\left(z_{j}^{-}\right)^{2 n}\left[\frac{\xi_{n}^{-}\left(z_{j}^{-}\right)}{\left(z-z_{j}^{-}\right)}+\frac{\xi_{n}^{-}\left(-z_{j}^{-}\right)}{\left(z+z_{j}^{-}\right)}\right], \\
& \xi_{n}^{-}(z)=\left(\begin{array}{l}
1 \\
0
\end{array}\right)+\sum_{j=1}^{S} C_{j}^{+}\left(z_{j}^{+}\right)^{-2 n}\left[\frac{\xi_{n}^{+}\left(z_{j}^{+}\right)}{\left(z-z_{j}^{+}\right)}+\frac{\xi_{n}^{+}\left(-z_{j}^{+}\right)}{\left(z+z_{j}^{+}\right)}\right] .
\end{aligned}
$$

Putting $z= \pm z_{j}^{ \pm}$in $(42 \mathrm{a})$ and $(42 \mathrm{~b})$, respectively gives

$$
\begin{aligned}
& \xi_{n}^{+}\left( \pm z_{j}^{+}\right)=\left(\begin{array}{l}
0 \\
1
\end{array}\right) \mp \sum_{k=1}^{S} C_{k}^{-}\left(z_{k}^{-}\right)^{2 n}\left[\frac{\xi_{n}^{-}\left(z_{k}^{-}\right)}{\left(z_{j}^{+} \mp z_{k}^{-}\right)}+\frac{\xi_{n}^{-}\left(-z_{k}^{-}\right)}{\left(z_{j}^{+} \pm z_{k}^{-}\right)}\right], \\
& \xi_{n}^{-}\left( \pm z_{j}^{-}\right)=\left(\begin{array}{l}
1 \\
0
\end{array}\right) \pm \sum_{k=1}^{S} C_{k}^{+}\left(z_{k}^{+}\right)^{-2 n}\left[\frac{\xi_{n}^{+}\left(z_{k}^{+}\right)}{\left(z_{j}^{-} \mp z_{k}^{+}\right)}+\frac{\xi_{n}^{+}\left(-z_{k}^{+}\right)}{\left(z_{j}^{-} \pm z_{k}^{+}\right)}\right],
\end{aligned}
$$

so, the above relations show that

$$
\begin{array}{ll}
\xi_{n}^{+, 1}\left(-z_{j}^{+}\right)=-\xi_{n}^{+, 1}\left(z_{j}^{+}\right), \quad \text { iff } \quad \xi_{n}^{-, 1}\left(-z_{j}^{-}\right)=\xi_{n}^{-, 1}\left(z_{j}^{-}\right), \\
\xi_{n}^{+, 2}\left(-z_{j}^{+}\right)=\xi_{n}^{+, 2}\left(z_{j}^{+}\right), \quad \text { iff } \quad \xi_{n}^{-, 2}\left(-z_{j}^{-}\right)=-\xi_{n}^{-, 2}\left(z_{j}^{-}\right) .
\end{array}
$$

Then it follows that the solution of $(42 \mathrm{a})$ and $(42 \mathrm{~b})$ reads

$$
\begin{aligned}
& \xi_{n}^{-, 1}\left(z_{1}^{-}\right)=\left[1-4 C_{1}^{+} C_{1}^{-} \frac{\left(z_{1}^{+}\right)^{-2(n-1)}\left(z_{1}^{-}\right)^{2 n}}{\left(\left(z_{1}^{+}\right)^{2}-\left(z_{1}^{-}\right)^{2}\right)^{2}}\right]^{-1}, \\
& \xi_{n}^{+, 2}\left(z_{1}^{+}\right)=\left[1+4 C_{1}^{+} C_{1}^{-} \frac{\left(z_{1}^{-}\right)^{2(n-1)}\left(z_{1}^{+}\right)^{-2 n}}{\left(\left(z_{1}^{+}\right)^{2}-\left(z_{1}^{-}\right)^{2}\right)^{2}}\right]^{-1} .
\end{aligned}
$$

Moreover, the potentials are given by

$$
\begin{aligned}
Q_{n-1}^{+} & =-2 C_{1}^{+}\left(z_{1}^{+}\right)^{-2 n-2} \xi_{n}^{+,(2)}\left(z_{1}^{+}\right), \\
Q_{n}^{-} & =2 C_{1}^{-}\left(z_{1}^{-}\right)^{2 n-2} \xi_{n}^{-,(1)}\left(z_{1}^{-}\right) .
\end{aligned}
$$

Then substituting equation (45) and (46) in (47) and (48) will give the general form of 1-soliton solution

$$
\begin{aligned}
& Q_{1 n}^{+}=-\frac{2 C_{1}^{-}\left(z_{1}^{-}\right)^{2 n}}{1+4 C_{1}^{+} C_{1}^{-}\left(\left(z_{1}^{+}\right)^{2}-\left(z_{1}^{-}\right)^{2}\right)^{(-2)}\left(z_{1}^{+}\right)^{-2 n}\left(z_{1}^{-}\right)^{2(n+1)}}, \\
& Q_{1 n}^{-}=\frac{2 C_{1}^{+}\left(z_{1}^{+}\right)^{-2(n+1)}}{1+4 C_{1}^{+} C_{1}^{-}\left(\left(z_{1}^{+}\right)^{2}-\left(z_{1}^{-}\right)^{2}\right)^{(-2)}\left(z_{1}^{+}\right)^{-2 n}\left(z_{1}^{-}\right)^{2(n+1)}} .
\end{aligned}
$$


Taking into account the time evolution of the normalisation constants:

$$
C_{1}^{+}(z, \tau)=C_{1}^{+}(0) e^{2 \mathrm{i} \omega_{1}^{+} \tau}, \quad C_{1}^{-}(z, \tau)=C_{1}^{-}(0) e^{-2 \mathrm{i} \omega_{1}^{-} \tau}
$$

where $\omega^{ \pm}=\frac{\mathrm{i}}{2}\left(z_{1}^{ \pm}-\left(z_{1}^{ \pm}\right)^{-1}\right)$ and the canonical symmetry condition $Q_{n}^{-}=-Q_{-n}^{*}$, one can reduce (49) and (50) into the standard form of the 1-soliton solution of nonlocal AL equation:

$$
Q_{1 n}^{+}=\frac{\left(\left(z_{1}^{+}\right)^{2}-\left(z_{1}^{-}\right)^{2}\right) e^{\mathrm{i} \alpha_{1}^{-}} e^{-2 \mathrm{i} \omega_{1}^{-} \tau}\left(z_{1}^{-}\right)^{2 n-1}}{1+\left(z_{1}^{-}\right)\left(z_{1}^{+}\right)^{-1} e^{\mathrm{i}\left(\alpha_{1}^{+}+\alpha_{1}^{-}\right)} e^{2 \mathrm{i}\left(\omega_{1}^{+}-\omega_{1}^{-}\right) \tau}\left(z_{1}^{+}\right)^{-2 n}\left(z_{1}^{-}\right)^{2 n}} .
$$

This reproduces the result obtained by M. Ablowitz and Z. Musslimani.

\section{$4.2 \quad 2-$ Soliton solution}

In a similar way as before (for the one pole) we will obtain the two-soliton solutions from a singular RHP with quartet of discrete eigenvalues/singularities $z_{\{1,2\}}^{+}$and $z_{\{1,2\}}^{-}$. The starting point here are the following linear integral equations for $\xi_{n}^{ \pm}(z)$ :

$$
\begin{aligned}
\xi_{n}^{+}(z)= & \left(\begin{array}{l}
0 \\
1
\end{array}\right)+\frac{1}{2 \pi \mathrm{i}} \oint_{\Omega}\left(\frac{\mathrm{d} \omega}{(\omega-z)}(\omega)^{2 n} \rho^{-}(\omega) \xi_{n}^{-}(\omega)\right)- \\
& {\left[C_{1}^{-}\left(z_{1}^{-}\right)^{2 n}\left(\frac{\xi_{n}^{-}\left(z_{1}^{-}\right)}{\left(z-z_{1}^{-}\right)}+\frac{\xi_{n}^{-}\left(-z_{1}^{-}\right)}{\left(z+z_{1}^{-}\right)}\right)\right]+\left[C_{2}^{-}\left(z_{2}^{-}\right)^{2 n}\left(\frac{\xi_{n}^{-}\left(z_{2}^{-}\right)}{\left(z-z_{2}^{-}\right)}+\frac{\xi_{n}^{-}\left(-z_{2}^{-}\right)}{\left(z+z_{2}^{-}\right)}\right)\right], \quad(52 \mathrm{a}) } \\
\xi_{n}^{-}(z)= & \left(\begin{array}{l}
1 \\
0
\end{array}\right)-\frac{1}{2 \pi \mathrm{i}} \oint_{\Omega}\left(\frac{\mathrm{d} \omega}{(\omega-z)}(\omega)^{-2 n} \rho^{+}(\omega) \xi_{n}^{+}(\omega)\right)+ \\
& {\left[C_{1}^{+}\left(z_{1}^{+}\right)^{-2 n}\left(\frac{\xi_{n}^{+}\left(z_{1}^{+}\right)}{\left(z-z_{1}^{+}\right)}+\frac{\xi_{n}^{+}\left(-z_{1}^{+}\right)}{\left(z+z_{1}^{+}\right)}\right)\right]+\left[C_{2}^{+}\left(z_{2}^{+}\right)^{-2 n}\left(\frac{\xi_{n}^{+}\left(z_{2}^{+}\right)}{\left(z-z_{2}^{+}\right)}+\frac{\xi_{n}^{+}\left(-z_{2}^{+}\right)}{\left(z+z_{2}^{+}\right)}\right)\right] . }
\end{aligned}
$$

The two-soliton solution again correspond to zero reflection coefficients (i.e. $\rho^{+}(z)=\rho^{-}(z)=0$ on $|z|=1$ ). In this case the system (52) reduces to a linear system of algebraic equations for $\xi_{n}^{+}(z)$

$$
\begin{aligned}
& \xi_{n}^{+}(z)=\left(\begin{array}{l}
0 \\
1
\end{array}\right)-\left[C_{1}^{-}\left(z_{1}^{-}\right)^{2 n}\left(\frac{\xi_{n}^{-}\left(z_{1}^{-}\right)}{\left(z-z_{1}^{-}\right)}+\frac{\xi_{n}^{-}\left(-z_{1}^{-}\right)}{\left(z+z_{1}^{-}\right)}\right)\right]- \\
& {\left[C_{2}^{-}\left(z_{2}^{-}\right)^{2 n}\left(\frac{\xi_{n}^{-}\left(z_{2}^{-}\right)}{\left(z-z_{2}^{-}\right)}+\frac{\xi_{n}^{-}\left(-z_{2}^{-}\right)}{\left(z+z_{2}^{-}\right)}\right)\right], }
\end{aligned}
$$

and a similar system for $\xi_{n}^{-}(z)$ :

$$
\begin{aligned}
& \xi_{n}^{-}(z)=\left(\begin{array}{l}
1 \\
0
\end{array}\right)+\left[C_{1}^{+}\left(z_{1}^{+}\right)^{-2 n}\left(\frac{\xi_{n}^{+}\left(z_{1}^{+}\right)}{\left(z-z_{1}^{+}\right)}+\frac{\xi_{n}^{+}\left(-z_{1}^{+}\right)}{\left(z+z_{1}^{+}\right)}\right)\right]+ \\
& {\left[C_{2}^{+}\left(z_{2}^{+}\right)^{-2 n}\left(\frac{\xi_{n}^{+}\left(z_{2}^{+}\right)}{\left(z-z_{2}^{+}\right)}+\frac{\xi_{n}^{+}\left(-z_{2}^{+}\right)}{\left(z+z_{2}^{+}\right)}\right)\right] . }
\end{aligned}
$$


Here $\xi_{n}^{+}\left( \pm z_{j}^{+}\right)$are the FAS $\xi_{n}^{+}(z)$ evaluated at the eigenvalue $\pm z_{j}^{+}$(similarly, $\xi_{n}^{-}\left( \pm z_{j}^{-}\right)$are the FAS $\xi_{n}^{-}(z)$ evaluated at the eigenvalue $\left.\pm z_{j}^{-}\right)$. We can find the expressions for these vectors by evaluating (53a) at the points $\pm z_{\{1,2\}}^{+}$and (53b) at the points $\pm z_{\{1,2\}}^{-}$. This results in a linear algebraic system composed of (53a) and (53b)

$$
\begin{aligned}
& \xi_{n}^{+}\left( \pm z_{1}^{+}\right)=\left(\begin{array}{l}
0 \\
1
\end{array}\right)-\left[C_{1}^{-}\left(z_{1}^{-}\right)^{2 n}\left(\frac{\xi_{n}^{-}\left(z_{1}^{-}\right)}{\left( \pm z_{1}^{+}-z_{1}^{-}\right)}+\frac{\xi_{n}^{-}\left(-z_{1}^{-}\right)}{\left( \pm z_{1}^{+}+z_{1}^{-}\right)}\right)\right]- \\
& {\left[C_{2}^{-}\left(z_{2}^{-}\right)^{2 n}\left(\frac{\xi_{n}^{-}\left(z_{2}^{-}\right)}{\left( \pm z_{1}^{+}-z_{2}^{-}\right)}+\frac{\xi_{n}^{-}\left(-z_{2}^{-}\right)}{\left( \pm z_{1}^{+}+z_{2}^{-}\right)}\right)\right]} \\
& \xi_{n}^{+}\left( \pm z_{2}^{+}\right)=\left(\begin{array}{l}
0 \\
1
\end{array}\right)-\left[C_{1}^{-}\left(z_{1}^{-}\right)^{2 n}\left(\frac{\xi_{n}^{-}\left(z_{1}^{-}\right)}{\left( \pm z_{2}^{+}-z_{1}^{-}\right)}+\frac{\xi_{n}^{-}\left(-z_{1}^{-}\right)}{\left( \pm z_{2}^{+}+z_{1}^{-}\right)}\right)\right]- \\
& {\left[C_{2}^{-}\left(z_{2}^{-}\right)^{2 n}\left(\frac{\xi_{n}^{-}\left(z_{2}^{-}\right)}{\left( \pm z_{2}^{+}-z_{2}^{-}\right)}+\frac{\xi_{n}^{-}\left(-z_{2}^{-}\right)}{\left( \pm z_{2}^{+}+z_{2}^{-}\right)}\right)\right]} \\
& \xi_{n}^{-}\left( \pm z_{1}^{-}\right)=\left(\begin{array}{l}
1 \\
0
\end{array}\right)+\left[C_{1}^{+}\left(z_{1}^{+}\right)^{-2 n}\left(\frac{\xi_{n}^{+}\left(z_{1}^{+}\right)}{\left( \pm z_{1}^{-}-z_{1}^{+}\right)}+\frac{\xi_{n}^{+}\left(-z_{1}^{+}\right)}{\left( \pm z_{1}^{-}+z_{1}^{+}\right)}\right)\right]+ \\
& {\left[C_{2}^{+}\left(z_{2}^{+}\right)^{-2 n}\left(\frac{\xi_{n}^{+}\left(z_{2}^{+}\right)}{\left( \pm z_{1}^{-}-z_{2}^{+}\right)}+\frac{\xi_{n}^{+}\left(-z_{2}^{+}\right)}{\left( \pm z_{1}^{-}+z_{2}^{+}\right)}\right)\right] \text {, }} \\
& \xi_{n}^{-}\left( \pm z_{2}^{-}\right)=\left(\begin{array}{l}
1 \\
0
\end{array}\right)+\left[C_{1}^{+}\left(z_{1}^{+}\right)^{-2 n}\left(\frac{\xi_{n}^{+}\left(z_{1}^{+}\right)}{\left( \pm z_{2}^{-}-z_{1}^{+}\right)}+\frac{\xi_{n}^{+}\left(-z_{1}^{+}\right)}{\left( \pm z_{2}^{-}+z_{1}^{+}\right)}\right)\right]+ \\
& {\left[C_{2}^{+}\left(z_{2}^{+}\right)^{-2 n}\left(\frac{\xi_{n}^{+}\left(z_{2}^{+}\right)}{\left( \pm z_{2}^{-}-z_{2}^{+}\right)}+\frac{\xi_{n}^{+}\left(-z_{2}^{+}\right)}{\left( \pm z_{2}^{-}+z_{2}^{+}\right)}\right)\right] \text {. }}
\end{aligned}
$$

From (54) and (55), it follows that

$$
\begin{array}{ll}
\xi_{n}^{+, 1}\left(-z_{j}^{+}\right)=-\xi_{n}^{+, 1}\left(z_{j}^{+}\right) \quad \text { iff } \quad \xi_{n}^{-, 1}\left(-z_{j}^{-}\right)=\xi_{n}^{-, 1}\left(z_{j}^{-}\right), \\
\xi_{n}^{+, 2}\left(-z_{j}^{+}\right)=\xi_{n}^{+, 2}\left(z_{j}^{+}\right) \quad \text { iff } \quad \xi_{n}^{-, 2}\left(-z_{j}^{-}\right)=-\xi_{n}^{-, 2}\left(z_{j}^{-}\right) .
\end{array}
$$

We can recover $Q_{n}^{-}$from the power series expansion of the RHS of $\xi_{n}^{-, 2}(z)$ in $(53 \mathrm{~b})$, and take the residue at $z \rightarrow z_{1}^{+}$or at $z \rightarrow z_{2}^{+}$, from which we then can obtain

$$
Q_{n}^{-}=-\frac{1}{2} C_{1}^{+}\left(z_{1}^{+}\right)^{-2 n-2} \xi_{n}^{+, 2}\left(z_{1}^{+}\right)+\frac{2 C_{2}^{+}\left(z_{2}^{+}\right)^{-2 n}}{\left(\left(z_{1}^{+}\right)^{2}-\left(z_{2}^{+}\right)^{2}\right)} \xi_{n}^{+, 2}\left(z_{2}^{+}\right),
$$

or

$$
Q_{n}^{-}=\frac{2 C_{1}^{+}\left(z_{1}^{+}\right)^{-2 n}}{\left(\left(z_{2}^{+}\right)^{2}-\left(z_{1}^{+}\right)^{2}\right)} \xi_{n}^{+, 2}\left(z_{1}^{+}\right)-\frac{1}{2} C_{2}^{+}\left(z_{2}^{+}\right)^{-2 n-2} \xi_{n}^{+, 2}\left(z_{2}^{+}\right)
$$

However it is difficult to find the potential from $\varphi_{n}^{-}(z)$. To fix the problem we will multiply the Laurent expansion function $\chi_{n}^{-}(z)$ by $\left(\begin{array}{cc}1 & 0 \\ 0 & c_{n}\end{array}\right)$ and compare it with RHS of (53a)

$$
\left(\tilde{\xi}_{n}^{-}, \tilde{\varphi}_{n}^{-}\right) \simeq \tilde{\chi}_{n}^{-}(z)=\left(\begin{array}{cc}
1 & 0 \\
0 & c_{n}
\end{array}\right)\left(\begin{array}{cc}
c_{n}^{-1} & z Q_{n-1}^{+} \\
c_{n}^{-1} z Q_{n}^{-} & 1
\end{array}\right)=\left(\begin{array}{cc}
c_{n}^{-1} & z Q_{n-1}^{+} \\
z Q_{n}^{-} & c_{n}
\end{array}\right)
$$


where $\tilde{\chi}_{n}^{-}(z)$ has the same power series expansions as $\chi_{n}^{-}(z)$. Then we can find the potential $Q_{n-1}^{+}$from $\tilde{\varphi}_{n}^{-, 1}$ in (58), since from $(37)$ (when $\left.b^{-}(z)=0\right)$

$$
\begin{aligned}
& \tilde{\varphi}_{n}^{-}(z)=\left(\begin{array}{l}
0 \\
1
\end{array}\right)-\left[C_{1}^{-}\left(z_{1}^{-}\right)^{2 n}\left(\frac{\xi_{n}^{-}\left(z_{1}^{-}\right)}{\left(z-z_{1}^{-}\right)}+\frac{\xi_{n}^{-}\left(-z_{1}^{-}\right)}{\left(z+z_{1}^{-}\right)}\right)\right]- \\
& {\left[C_{2}^{-}\left(z_{2}^{-}\right)^{2 n}\left(\frac{\xi_{n}^{-}\left(z_{2}^{-}\right)}{\left(z-z_{2}^{-}\right)}+\frac{\xi_{n}^{-}\left(-z_{2}^{-}\right)}{\left(z+z_{2}^{-}\right)}\right)\right] . }
\end{aligned}
$$

We have a quartet of eigenvalues $\pm z_{j}^{+}, \pm z_{j}^{-}$with $\left|z_{j}^{+}\right|>1$ and $\left|z_{j}^{-}\right|<1$ respectively, thus we can solve the linear algebra system (54a)-(55b) for $\xi_{n}^{-}\left(z_{1}^{-}\right), \xi_{n}^{-}\left(z_{2}^{-}\right), \xi_{n}^{+}\left(z_{1}^{+}\right)$and $\xi_{n}^{+}\left(z_{2}^{+}\right)$. In particular, in order to find $Q_{n}^{+}$we will need $\xi_{n}^{-}\left(z_{1}^{-}\right)$and $\xi_{n}^{-}\left(z_{2}^{-}\right)$:

$$
\begin{aligned}
& \xi_{n}^{+, 1}\left(z_{1}^{+}\right)=-2 z_{1}^{+}\left[\frac{C_{1}^{-}\left(z_{1}^{-}\right)^{2 n}}{\left(\left(z_{1}^{+}\right)^{2}-\left(z_{1}^{-}\right)^{2}\right)} \xi_{n}^{-, 1}\left(z_{1}^{-}\right)+\frac{C_{2}^{-}\left(z_{2}^{-}\right)^{2 n}}{\left(\left(z_{1}^{+}\right)^{2}-\left(z_{2}^{-}\right)^{2}\right)} \xi_{n}^{-, 1}\left(z_{2}^{-}\right)\right] \\
& \xi_{n}^{+, 1}\left(z_{2}^{+}\right)=-2 z_{2}^{+}\left[\frac{C_{1}^{-}\left(z_{1}^{-}\right)^{2 n}}{\left(\left(z_{2}^{+}\right)^{2}-\left(z_{1}^{-}\right)^{2}\right)} \xi_{n}^{-, 1}\left(z_{1}^{-}\right)+\frac{C_{2}^{-}\left(z_{2}^{-}\right)^{2 n}}{\left(\left(z_{2}^{+}\right)^{2}-\left(z_{2}^{-}\right)^{2}\right)} \xi_{n}^{-, 1}\left(z_{2}^{-}\right)\right], \\
& \xi_{n}^{-, 1}\left(z_{1}^{-}\right)=1+\frac{2 C_{1}^{+}\left(z_{1}^{+}\right)^{-2(n-1)}}{\left(\left(z_{1}^{-}\right)^{2}-\left(z_{1}^{+}\right)^{2}\right)} \xi_{n}^{+, 1}\left(z_{1}^{+}\right)+\frac{2 C_{2}^{+}\left(z_{2}^{+}\right)^{-2(n-1)}}{\left(\left(z_{1}^{-}\right)^{2}-\left(z_{2}^{+}\right)^{2}\right)} \xi_{n}^{+, 1}\left(z_{2}^{+}\right), \\
& \xi_{n}^{-, 1}\left(z_{2}^{-}\right)=1+\frac{2 C_{1}^{+}\left(z_{1}^{+}\right)^{-2(n-1)}}{\left(\left(z_{2}^{-}\right)^{2}-\left(z_{1}^{+}\right)^{2}\right)} \xi_{n}^{+, 1}\left(z_{1}^{+}\right)+\frac{2 C_{2}^{+}\left(z_{2}^{+}\right)^{-2(n-1)}}{\left(\left(z_{2}^{-}\right)^{2}-\left(z_{2}^{+}\right)^{2}\right)} \xi_{n}^{+, 1}\left(z_{2}^{+}\right),
\end{aligned}
$$

Now, by comparing the power series expansion of the RHS of (59) to the expansion (58), we obtain the potential when $z \rightarrow z_{1}^{-}$or $z \rightarrow z_{2}^{-}$

$$
Q_{n-1}^{+}=\frac{1}{2} C_{1}^{-}\left(z_{1}^{-}\right)^{2 n-2} \xi_{n}^{-, 1}\left(z_{1}^{-}\right)-\frac{2 C_{2}^{-}\left(z_{2}^{-}\right)^{2 n}}{\left(\left(z_{1}^{-}\right)^{2}-\left(z_{2}^{-}\right)^{2}\right)} \xi_{n}^{-, 1}\left(z_{2}^{-}\right) .
$$

or

$$
Q_{n-1}^{+}=\frac{-2 C_{1}^{-}\left(z_{1}^{-}\right)^{2 n}}{\left(\left(z_{2}^{-}\right)^{2}-\left(z_{1}^{-}\right)^{2}\right)} \xi_{n}^{-, 1}\left(z_{1}^{-}\right)+\frac{1}{2} C_{2}^{-}\left(z_{2}^{-}\right)^{2 n-2} \xi_{n}^{-, 1}\left(z_{2}^{-}\right) .
$$

Then substituting each (60c) and (60d), in (61) or (62) and using the involution (4), with negative sign, then equation (61) is the solution for the nonlocal discrete NLS equation.

\section{Spectral properties of $L_{n}(z)$ and completeness of the Jost solutions}

The crucial fact that determines the spectral properties of the operator $L_{n}(z)$ is the choice of the class of functions where from we shall choose the potential $Q(x)$. Here we assume that $Q_{n}(t)$ is a differentiable function for all $t \in \mathbb{R}$ and exists for all $n \in \mathbb{Z}$. In additional, we assume that it tends to zero as $n \rightarrow \pm \infty$.

The FAS $\chi_{n}^{ \pm}(z)$ of $L_{n}(z)$ allow one to construct the resolvent $R_{n, m}(z)$ of the operator $L_{n}(z)$ and then to investigate its spectral properties. From the general theory of linear operators we know that the point in the complex $z$-plane is a regular point if $R_{n, m}(z)$ is a bounded integral 
operator. In each connected subset of regular points $R(z)$ is analytic in $z$. The points which are not regular constitute the spectrum of $L_{n}(z)$ : the continuous spectrum of $L_{n}(z)$ consists of all points $z$ for which $R_{n, m}(z)$ is an unbounded integral operator while the discrete spectrum of $L_{n}(z)$ consists of all points $z$ for which $R_{n, m}(z)$ develops pole singularities.

The kernel of the resolvent $R_{n, m}(z)$ can be expressed through the FAS of $\chi_{n}^{ \pm}(z)$ as follows:

$$
\begin{aligned}
& R_{\{n, m\}}^{+}(z)=\chi_{n+1}^{+}(z)\left(\begin{array}{cc}
\theta(m-n) & 0 \\
0 & \theta(n-m)
\end{array}\right) \hat{\chi}_{m}^{+}(z), \\
& R_{\{n, m\}}^{-}(z)=\chi_{n+1}^{-}(z)\left(\begin{array}{cc}
\theta(n-m) & 0 \\
0 & \theta(m-n)
\end{array}\right) \hat{\chi}_{m}^{-}(z) .
\end{aligned}
$$

Note that by construction, $R_{\{n, m\}}^{ \pm}(z)$ is analytic in $\gamma^{ \pm}$respectively.

We will derive the completeness relation for the Jost solutions of $L_{n}$, by construction the partition of unity of the group of fundamental solutions of $L_{n}(z)$. For this purpose, we will use again the contour integration method for suitably chosen contours which do not cross the continuous spectrum of $L_{n}(z)$. We need to evaluate the integral

$$
\mathcal{J}_{R,\{n, m\}}(z)=\frac{1}{2 \pi \mathrm{i}}\left(\oint_{\gamma^{+}} \mathrm{d} z R_{n}^{+}(z)-\oint_{\gamma^{-}} \mathrm{d} z R_{n}^{-}(z)\right),
$$

along the contours $\gamma_{ \pm}$, as shown on Figure 1. According to Cauchy's residue theorem, one has

$$
\mathcal{\partial}_{R,\{n, m\}}(z)=\sum_{j=1}^{N}\left(\operatorname{Res}_{z= \pm z_{j}^{+}} R_{n}^{+}(z)+\operatorname{Res}_{z= \pm z_{j}^{-}} R_{n}^{-}(z)\right) .
$$

Here we denoted by $z_{j}^{ \pm}$the discrete eigenvalues of $L_{n}(z)$ that are outside/inside the unit circle $|z|=1$, respectively. We will again assume that their numbers are equal and that all of them are isolated singularities for the resolvent.

Each of the integrals in (64) can be written as a sum of integral over the continuous spectrum $\Omega$ and an integral involving asymptotics for $|z| \rightarrow \infty$ and $|z| \rightarrow 0$ :

$$
\frac{1}{2 \pi \mathrm{i}} \oint_{\gamma^{ \pm}} \mathrm{d} z R_{n}^{ \pm}(z)=\int_{|z|=1} \mathrm{~d} z R_{n}^{ \pm}(z)-\oint_{\gamma_{\text {as }}^{ \pm}} \mathrm{d} z R_{n}^{ \pm}(z)
$$

First, in order to find the residues in (65) one needs the Laurent series expansions of the FAS and scattering data around the points of the discrete spectrum $z_{j}^{ \pm}$. Using (20), one can write the following expansions:

$$
\begin{aligned}
& a^{ \pm}(z)=\left(z-\left( \pm z_{j}^{ \pm}\right)\right) \dot{a}_{j}^{ \pm}+\frac{1}{2}\left(z-\left( \pm z_{j}^{ \pm}\right)\right)^{2} \ddot{a}_{j}^{ \pm}+\ldots, \\
& \alpha^{ \pm}(z)=\left(z-\left( \pm z_{j}^{ \pm}\right)\right) \dot{\alpha}_{j}^{ \pm}+\frac{1}{2}\left(z-\left( \pm z_{j}^{ \pm}\right)\right)^{2} \ddot{\alpha}_{j}^{ \pm}+\ldots, \\
& \chi_{n}^{+}\left(z_{j}^{+}\right)=\psi_{n, j}^{+}(z)\left(b_{j}^{+}, 1\right)=\phi_{n, j}^{+}(z)\left(1,1 / b_{j}^{+}\right), \\
& \hat{\chi}_{n}^{+}\left(z_{j}^{+}\right)=\left(\begin{array}{c}
1 \\
-\beta_{j}^{+}
\end{array}\right) \frac{\tilde{\Psi}_{n, j}^{+}(z)}{\left(z- \pm z_{j}^{+}\right) \dot{\alpha}_{j}^{+}}=\left(\begin{array}{c}
1 / \beta_{j}^{+} \\
-1
\end{array}\right) \frac{\tilde{\Phi}_{n, j}^{+}(z)}{\left(z- \pm z_{j}^{+}\right) \dot{\alpha}_{j}^{+}}, \\
& \chi_{n}^{-}\left(z_{j}^{-}\right)=\psi_{n, j}^{-}(z)\left(1,-b_{j}^{-}\right)=\phi_{n, j}^{-}(z)\left(-1 / b_{j}^{-}, 1\right), \\
& \hat{\chi}_{n}^{-}\left(z_{j}^{-}\right)=-\left(\begin{array}{c}
\beta_{j}^{-} \\
1
\end{array}\right) \frac{\tilde{\Psi}_{n, j}^{-}(z)}{\left(z- \pm z_{j}^{-}\right) \dot{\alpha}_{j}^{-}}=\left(\begin{array}{c}
1 \\
1 / \beta_{j}^{-}
\end{array}\right) \frac{\tilde{\Phi}_{n, j}^{-}(z)}{\left(z- \pm z_{j}^{-}\right) \dot{\alpha}_{j}^{-}},
\end{aligned}
$$


where $\tilde{\Psi}_{n}(z)$ and $\tilde{\Phi}_{n}(z)$ are related with $\psi_{n}(z)$ and $\phi_{n}(z)$ respectively. As a result, one can find the residues of $R_{n}^{ \pm}(z)$ at $z= \pm z_{j}^{ \pm}$:

$$
\operatorname{Res}_{z= \pm z_{j}^{ \pm}} R_{n, m}^{ \pm}(z)=\mp \frac{\phi_{n+1, j}^{ \pm}(z) \tilde{\Psi}_{m, j}^{ \pm}(z)}{\dot{\alpha}_{j}^{ \pm}(z)} .
$$

Now, we can calculate the jump of $R_{n}(z)$ on the unite circle $(|z|=1)$. Using (63a) one can obtain the result

$$
\int_{|z|=1} \mathrm{~d} z\left(R_{n, m}^{+}(z)-R_{n, m}^{-}(z)\right)=\int_{|z|=1} \mathrm{~d} z\left(\frac{\phi_{n+1}^{+}(z) \tilde{\Psi}_{m}^{+}(z)}{\alpha^{+}(z)}+\frac{\phi_{n+1}^{-}(z) \tilde{\Psi}_{m}^{-}(z)}{\alpha^{-}(z)}\right) .
$$

Finally, in order to calculate the contribution of the integrals over the asymptotic circles (i.e when $|z| \rightarrow \infty$ and $|z| \rightarrow 0$, respectively). For this purpose we need the asymptotics of FAS $\chi_{n, a s}^{ \pm}(z)$ for $z \rightarrow \infty$ and $z \rightarrow 0$ :

$$
\chi_{\{\mathrm{as}, n\}}^{+}(z)=\left(\begin{array}{cc}
z^{n} & 0 \\
0 & z^{-n}
\end{array}\right)+\mathcal{O}(1 / z), \quad z \rightarrow \infty ; \quad \chi_{\{\mathrm{as}, n\}}^{-}(z)=\left(\begin{array}{cc}
z^{n} & 0 \\
0 & z^{-n}
\end{array}\right)+\mathcal{O}(z), \quad z \rightarrow 0 .
$$

A direct contour integration shows (if we take a limit $|z| \rightarrow \infty$ in the integral over $\gamma_{\text {as }}^{-}$and a limit $|z| \rightarrow 0$ in the integral over $\gamma_{\text {as }}^{+}$, respectively) that

$$
\frac{1}{2 \pi \mathrm{i}}\left(\oint_{\gamma_{\text {as }}^{+}} \mathrm{d} z R_{\{\text {as }, n, m\}}^{+}+\oint_{\gamma_{\text {as }}^{-}} \mathrm{d} z R_{\{\text {as }, n, m\}}^{-}\right)=\delta(n-m) \mathbb{I} .
$$

As a result, combining (64) and (65) and taking into account (68), (69) and (70), one gets the following completeness relation:

$$
\begin{aligned}
\delta(n-m) \mathbb{1}= & \frac{1}{2 \pi \mathrm{i}} \int_{|z|=1} \mathrm{~d} z\left(\frac{\phi_{n+1}^{+}(z) \tilde{\Psi}_{m}^{+}(z)}{\alpha^{+}(z)}+\frac{\phi_{n+1}^{-}(z) \tilde{\Psi}_{m}^{-}(z)}{\alpha^{-}(z)}\right) \\
& +\sum_{j=1}^{N}\left(\frac{\phi_{\{n+1, j\}}^{+}(z) \tilde{\Psi}_{m, j}^{+}(z)}{\dot{\alpha}_{j}^{+}(z)}-\frac{\phi_{\{n+1, j\}}^{-}(z) \tilde{\Psi}_{m, j}^{-}(z)}{\dot{\alpha}_{j}^{-}(z)}-\right) .
\end{aligned}
$$

Therefore, the Jost solutions $\phi_{n}^{ \pm}(z)$ form a complete set of functions over the space of fundamental solutions of $L_{n}(z)$.

Based on the completeness relation (71), one can expand every function $Y(x)$ from the space of solutions of $L_{n}(z)$ over the complete set Jost solutions by the following expansion formulae:

$$
\begin{aligned}
Y(x)= & \frac{1}{2 \pi \mathrm{i}} \int_{|z|=1} \mathrm{~d} z\left(\phi_{n+1}^{+}(z) y_{m}^{+}(z)+\phi_{n+1}^{-}(z) y_{m}^{-}(z)\right) \\
& +\sum_{j=1}^{N}\left(\phi_{\{n+1, j\}}^{+}(z) y_{\{m, j\}}^{+}(z)-\phi_{\{n+1, j\}}^{-}(z) y_{\{m, j\}}^{-}(z)\right),
\end{aligned}
$$

where

$$
\begin{aligned}
& y_{m}^{ \pm}(z)=\frac{1}{\alpha^{ \pm}(z)} \int_{|z|=1} \mathrm{~d} z \tilde{\Psi}_{m}^{ \pm}(z) Y_{m}(z) \\
& y_{\{m, j\}}^{ \pm}=\frac{1}{\dot{\alpha}_{j}^{ \pm}} \int_{|z|=1} \mathrm{~d} z \tilde{\Psi}_{\{m, j\}}^{ \pm}(z) Y_{m}(z) .
\end{aligned}
$$




\section{Conclusions}

We have studied here a nonlocal version [10] of the semi-discrete NLS equation in the AblowitzLadik form. This equation appears to be $\mathcal{P} \mathcal{T}$ symmetric. We formulated the direct scattering problem for the nonlocal Ablowitz-Ladik equation. This incudes: the construction of the Jost solutions and the minimal set of scattering data, the construction of the fundamental analytic solutions (FAS). Then, based on the formulation of the inverse scattering transform for (4) in the form of additive Riemann-Hilbert boundary value problem, the 1- and 2-soliton solutions are derived.

It was shown in [10] that the 1-soliton solution develops a singularity in finite time. This was due to the disbalance of the associated Riemann-Hilbert problem - the numbers of zeroes of the FAS inside the boundary contour is not equal to the number of zeroes inside the contour: the nonlocal involution requires that if $z_{j}$ is a discrete eigenvalue then $z_{j}^{*}$ must me also an eigenvalue, i.e. both $z_{j}$ and $z_{j}^{*}$ must be either inside or outside the unit circle. Depending on the positions of the discrete eigenvalues $z_{j}^{ \pm}$in the spectral plane, there are two regimes for the 2 -soliton solution: if one of the discrete eigenvalues is inside the unit circle and the other is outside, then the nonlocal involution will preserve their number inside and outside the contour balanced and as a result, the corresponding 2-soliton solutions will be regular for all $t$. Otherwise, the 2-soliton solution will develop again a singularity in finite time.

Finally, we outlined briefly the spectral properties of the Lax operator $L_{n}(z)$. We have derived the completeness relations for the Jost solutions and obtained expansions over the complete set of Jost solutions for a generic function from the space of solutions of $L_{n}(z)$.

The results of this paper can be extended in several directions:

- To construct gauge covariant formulation of the inverse scattering method for nonlocal Ablowitz-Ladik equation (49), including the generating (recursion) operator [38] and it spectral decomposition [26], the description of the class of the differential-difference equations solvable by the spectral problem (6) (i.e. the corresponding integrable hierarchy), the description of the infinite set of integrals of motion and the hierarchy of Hamiltonian structures.

- To study the gauge equivalent systems [29,32,33].

- To study the inverse scattering method for the equivalent spectral eigenvalue problem (12). In this case the corresponding Riemann-Hilbert problem is with a canonical normalisation.

- To study the associated Darboux transformations and their generalizations for both local and nonlocal Ablowitz-Ladik equations. This will provide an algebraic method for constructing and classification of possible soliton solutions, including also rational solutions [20].

- To extend the results of this paper for the case of non-vanishing boundary conditions (a non-trivial background) $[12,46,47,52]$. In the local case, such solutions are of interest in nonlinear optics: they arise in the theory of ultrashort femto-second nonlinear pulses in optical fibers. The nonlocal reduction of the Ablowitz-Ladik equation can be of particular interest in the theory of electromagnetic waves in artificial heterogenic media [64]. The considerations required in this case are more complicated and will be discussed elsewhere.

- To study multi-component generalisations [27, 28,41,42,43] for both local and nonlocal semi-discrete NLS equation. This includes the block Ablowitz-Ladik system [35] and generalisations to homogeneous and symmetric spaces. Such multi-component generalisations 
are much more complicated compared to the continuous case and, to the best of our knowledge, they were not studied up till now.

\section{Acknowledgements}

The authors have the pleasure to thank Prof. Mark Ablowitz and Prof. Vladimir Gerdjikov for numerous useful discussions.

\section{References}

[1] F.K. Abdullaev, Y.V. Kartashov, V.V. Konotop, D.A. Zezyulin, Solitons in PT-symmetric nonlinear lattices, Phys. Rev. A 83 (2011), 041805.

[2] Ablowitz M. J. and Ladik J. F., Nonlinear differential-difference equations, J. Math. Phys. 16 (1975) 598-603.

[3] Ablowitz M. J. and Ladik J. F., Nonlinear differential-difference equations and Fourier Analysis, J. Math. Phys. 17 (1976) 1011-1018.

[4] Ablowitz M. J. and Ladik J. F., A nonlinear difference scheme and inverse scattering, Stud. Appl. Math. 55 (1976) 213-229.

[5] M. J. Ablowitz, B. Prinari and D. A. Trubach, Discrete and Continuous Nonlinear Schrödinger Systems, LMS Lecture Note Series 302, Cambridge University Press, Cambridge (2004).

[6] M.J. Ablowitz, B. Prinari and A.D. Trubatch, Discrete Vector Solitons: Composite Solitons, Yang-Baxter Maps and Computation, Stud. App. Math. 116 (2006), 97-133.

[7] M.J. Ablowitz, G. Biondini and B. Prinari, Inverse scattering transform for the integrable discrete nonlinear Schrödinger equation with non-vanishing boundary conditions, Inv. Probl. 23 (2007), 1711-1758.

[8] Ablowitz M. J., Ohta Y., Trubatch A. D., On discretizations of the vector nonlinear Schrdinger equation, Phys. Lett. A 253 (1999) 287-304.

[9] M. Ablowitz and Z. Musslimani, Integrable Nonlocal Nonlinear Schrödinger Equation, Phys. Rev. Lett., 110 (2013) 064105.

[10] M. Ablowitz and Z. Musslimani, Integrable discrete PT symmetric model, Phys. Rev. E 90 (2014) 032912.

[11] MJ Ablowitz, ZH Musslimani, Inverse scattering transform for the integrable nonlocal nonlinear Schrdinger equation, Nonlinearity 29 (2016), 915-946.

[12] MJ Ablowitz, H-D Luo, ZH Musslimani, Inverse scattering transform for the nonlocal nonlinear Schrödinger equation with nonzero boundary conditions, E-print: arXiv:1612.02726.

[13] VA Atanasov, VS Gerdjikov, GG Grahovski, NA Kostov, Fordy-Kulish model and spinor Bose-Einstein condensate, J. Nonl. Math. Phys. 15 (2008), 291-298. 
[14] I. V. Barashenkov, Hamiltonian formulation of the standard PT-symmetric nonlinear Schrödinger dimer, Physical Review A 90 (2014) 045802.

[15] C. M. Bender and S. Boettcher, Real Spectra in Non-hermitian Hamiltonians Having PT Symmetry, Phys. Rev. Lett 80 (1998) 5243-5246;

C. M. Bender, S. Boettcher and P. N. Meisinger, PT-Symmetric quantum Mechanics, J. Math. Phys. 40 (1999) 2201-2229.

[16] C. M. Bender, Making Sense of Non-hermitian Hamiltonians, Rep. Progr. Phys. 70 (2007) 947-1018 (E-print: hep-th/0703096).

[17] G. Biondini and A. Bui, The Ablowitz-Ladik system with linearizable boundary conditions, J. Phys. A: Math. Theor. 48 (2015) 375202 (31pp).

[18] Bruschi M., Manakov S. V., Ragnisco O., and Levi D., The nonabelian Toda lattice - Discrete analog of the matrix Schrödinger spectral problem, J. Math. Phys. 21 (1980) 2749-2753.

[19] Bruschi M., Manakov S. V., Ragnisco O., and Levi D., Evolution equations associated with the discrete analog of the matrix Schrödinger spectral problem solvable by IST, J. Math. Phys. 22 (1981) 2463-2471.

[20] E. V. Doktorov, and S. B. Leble, Dressing method in Mathematical Physics, Mathematical Physics Studies 28, Springer, Berlin, Heidelberg, New York (2007).

[21] E. V. Doktorov, N. P. Matsuka and V. M. Rothos, Perturbation-induced radiation by the Ablowitz-Ladik soliton, Phys. Rev. E 68 (2003) 066610 (14 pp.).

[22] E. V. Doktorov, N. P. Matsuka and V. M. Rothos, Dynamics of the Ablowitz-Ladik soliton train, Phys. Rev. E 69 (2004), 056607 (7 pp.).

[23] L. D. Faddeev, L. A. Takhtadjan, Hamiltonian Method in the Theory of Solitons, Springer Verlag, Berlin (1987).

[24] A Fring, $\mathcal{P T}$-symmetric deformations of integrable models, Phil. Trans. Royal Soc. A 371 (2013), 20120046.

[25] V. S. Gerdjikov, Generalised Fourier transforms for the soliton equations. Gauge covariant formulation, Inverse Problems 2 (1986), 51-74.

[26] V. S. Gerdjikov and G. G. Grahovski, Multi-component NLS models on symmetric spaces: Spectral properties versus representations theory, SIGMA 6 (2010), paper 044 (29 pages).

[27] V. S. Gerdjikov, G. G. Grahovski, N. A. Kostov, Reductions of N-wave interactions related to low-rank simple Lie algebras. I: $\mathbb{Z}_{2}$ - reductions, J. Phys. A: Math \& Gen. 34 (2001) 9425-9461 (E-print: nlin.SI/0006001).

[28] V. S. Gerdjikov, G. G. Grahovski, R. I. Ivanov, N. A. Kostov, N-wave interactions related to simple Lie algebras. $\mathbb{Z}_{2}$ - reductions and soliton solutions, Inverse Problems 17 (2001) 999-1015 (E-print: nlin.SI/0009034).

[29] V. S. Gerdjikov, G. G. Grahovski, N. A. Kostov, On N-wave Type Systems and Their Gauge Equivalent, European J. Phys. B 29 (2002) 243-248. 
[30] V. S. Gerdjikov, G. G. Grahovski and R. I. Ivanov, On the N-wave Equations with $\mathcal{P} \mathcal{T}$ symmetry, Theor. Math. Phys. 188 (2016), 1305-1321.

[31] V. S. Gerdjikov, G. G. Grahovski and R. I. Ivanov, On integrable wave interactions and Lax pairs on symmetric spaces, Wave Motion 71 (2017), 53-70.

[32] V. S. Gerdjikov, G. G. Grahovski, A. V. Mikhailov, T. I. Valchev, Rational bundles and recursion operators for integrable equations on A. III-type symmetric spaces, Theor. Math. Phys. 167 (2011), 740-750.

[33] V. S. Gerdjikov, G. G. Grahovski, A. V. Mikhailov, T. I. Valchev, Polynomial bundles and generalised Fourier transforms for integrable equations on A. III-type symmetric spaces, SIGMA 7 (2011), paper 096 (48 pages).

[34] V. S. Gerdjikov and M. I. Ivanov, Hamiltonian structure of multi-component nonlinear Schrödinger equations in difference form, Theor. Math. Phys. 52 (1982), 676-685.

[35] V. S. Gerdjikov and M. I. Ivanov, Block discrete Zakharov-Shabat system I: Generalized Fourier expansions, Commun. JINR E2-81-811 (1981), Dubna (USSR);

V. S. Gerdjikov and M. I. Ivanov, Block discrete Zakharov-Shabat system II: Hamiltonian structures, Commun. JINR E2-81-812 (1981)

[36] V. S. Gerdjikov, M. I. Ivanov and P. P. Kulish, Complete integrability of the difference evolution equations, Commun. JINR E2-80-882, (1980), Dubna (USSR).

[37] V. S. Gerdjikov, M. I. Ivanov and P. P. Kulish, Expansions over the "squared" solutions and difference evolution equations, J. Math. Phys. 25 (1984), 25-34.

[38] V. S. Gerdjikov, P. P. Kulish, The generating operator for the $n \times n$ linear system, Physica D 3 (1981) 549-564.

[39] V. S. Gerdjikov, A. Saxena, Complete Integrability of Nonlocal Nonlinear Schrödinger equation, J. Math. Phys. 58 (2017), 013502 (E-print: arXiv: 1510.0480).

[40] V. S. Gerdjikov, G. Vilasi, and A. B. Yanovski, Integrable Hamiltonian Hierarchies. Spectral and Geometric Methods, Lecture Notes in Physics 748, Springer Verlag, Berlin, Heidelberg, New York (2008).

[41] G. G. Grahovski, On the Reductions and Scattering Data for the Generalized ZakharovShabat Systems, In: "Nonlinear Physics: Theory and Experiment. II", Eds: M. J. Ablowitz, M. Boiti, F. Pempinelli and B. Prinari, World Scientific, Singapore (2003), pp. 71-78; G. G. Grahovski, Condon M., On the Caudrey-Beals-Coifman System and the Gauge Group Action, J. Nonlin. Math. Phys. 15 (2008), suppl. 3, 197-208 (E-print: arXiv:0710.3302).

[42] M. Gürses, Nonlocal Fordy-Kulish equations on symmetric spaces, Phys. Lett. A 381 (2017), 1791-1794.

[43] Fordy A.P., Kulish P.P., Nonlinear Schrödinger equations and simple Lie algebras, Comm. Math. Phys. 89 (1983), 427-443.

[44] Y. Ishimori, An integrable classical spin chain, J. Phys. Soc. Japan 51 (1982), 3417-3418. 
[45] V. M. Kenkre and D. K. Campbell, Self-trapping on a dimer: time-dependent solutions of a discrete nonlinear Schrödinger equation, Phys. Rev. B 34 (1986), 4959-61.

[46] Min Li and Tao Xu, Dark and antidark soliton interactions in the nonlocal nonlinear Schrödinger equation with the self-induced parity-time-symmetric potential, Phys. Rev. E 91 (2015), 033202.

[47] Min Li, Tao Xu and Dexin Meng, Rational Solitons in the Parity-Time-Symmetric Nonlocal Nonlinear Schrödinger Model, J. Phys. Soc. Japan 85 (2016), 124001.

[48] A. Mostafazadeh, Pseudo-hermiticity versus $\mathcal{P} \mathcal{T}$-Symmetry I, II, III, J. Math. Phys. 43 (2002) 205-214 (E-print: math-ph/0107001); 2814-2816 (E-print: math-ph/0110016); 3944-3951 (E-print: math-ph/0203005).

[49] A. Mostafazadeh, Pseudo-hermiticity and Generalized $\mathcal{P} \mathcal{T}$ - and $\mathcal{C P T}$-Symmetries, J. Math. Phys. 44 (2003) 974-989 (E-print: math-ph/0209018);

A. Mostafazadeh, Exact PT-Symmetry Is Equivalent to Hermiticity, J. Phys. A: Math. Gen. 36 (2003) 7081-7091 (E-print: quant-ph/0304080).

[50] A. V. Mikhailov, The reduction problem and the inverse scattering problem, Physica D 3 (1981) 73-117.

[51] N. Papanicoulau, Complete integrability for a discrete Heisenberg chain, J. Phys. A: Math. Gen. 20 (1987), 3637-3652.

[52] B. Prinari and F. Vitale, Inverse scattering transform for the focusing Ablowitz-Ladik system with nonzero boundary conditions, Stud. App. Math. 137 (2016), 28-52.

[53] Tsuchida T., Ujino H., and Wadati M., Integrable semi-discretization of the coupled nonlinear Schrödinger equations, J. Phys. A: Math. Gen. 32 (1999) 2239-2262.

[54] C. E. Rüter, K. G. Makris, R. El-Ganainy, D. N. Christodoulides, M. Segev and D. Kip, Observation of parity-time symmetry in optics, Nature Physics 6 (2010) 192 - 195.

[55] Shabat A. B., Inverse scattering problem for a system of differential equations, Functional Annal. \& Appl. 9 (1975), 75-78 (In Russian);

Shabat A. B. The inverse scattering problem, Diff. Equations 15 (1979), 1824-1834.

[56] S. Takeno and K. Hori, A propagating self-localized mode in a one-dimensional lattice with quartic anharmonicity, J. Phys. Soc. Japan 59 (1990), 3037-40.

[57] T. I. Valchev, On Mikhailov's reduction group, Phys. Lett A 379 (2015) 1877-1880.

[58] V. E. Zakharov, Exact solutions of the problem of parametric interaction of wave packets, Dokl. Akad. Nauk SSSR 228 (1976), no. 6, 1314-1316.

[59] S. P. Novikov, S. V. Manakov, L. P. Pitaevskii, and V. E. Zakharov, Theory of solitons: the inverse scattering method, New York: Plenum Press (1984).

[60] V. E. Vekslerchik and V. V. Konotop, Discrete nonlinear Schrödinger equation under non-vanishing boundary conditions, Inv. Problems 8 (1992) 889-909.

[61] V. E. Vekslerchik, Finite nonlinear Schrödinger chain, Phys. Lett. A 174 (1993), 285-288. 
[62] V. E. Vekslerchik, Functional representation of the Ablowitz-Ladik hierarchy I, J. Phys. A: Math. Gen. 31 (1998) 1087-1099;

V. E. Vekslerchik, Functional representation of the Ablowitz-Ladik hierarchy II, J. Nonlin. Math. Phys. 9 (2002), 157-180.

[63] V. E. Zakharov, and A. B. Shabat, A scheme for integrating nonlinear evolution equations of mathematical physics by the inverse scattering method. I $\&$ II, Funkts. Anal. Prilozhen., 8 (1974), 43-53; 13 (1979) no. 3, 13-22.

[64] A. A. Zyablovsky, A. P. Vinogradov, A. A. Pukhov, A. V. Dorofeenko and A A Lisyansky, PT-symmetry in optics, Phys.-Uspekhi 57 (2014), no. 11, 1063-1082. 